\title{
1 Measuring individual identity information in animal signals: 2 Overview and performance of available identity metrics 3
}

4 Pavel Linhart ${ }^{1}$, Tomasz Osiejuk ${ }^{1}$, Michal Budka ${ }^{1}$, Martin Šálek ${ }^{2,3}$, Marek Špinka ${ }^{4}$, Richard Policht ${ }^{4,5}$, 5 Michaela Syrová ${ }^{4,6}$, Daniel T. Blumstein ${ }^{7,8}$

6

7 Affiliations:

81 Department of Behavioural Ecology, Adam Mickiewicz University, Umultowska 89, 61-614, Poznan,

9 Poland

102 The Czech Academy of Sciences, Institute of Vertebrate Biology, Květná 8, 60365 Brno, Czech

11 Republic

123 Faculty of Environmental Sciences, Czech University of Life Sciences Prague, Kamýcká 1176,

13 Suchdol, 16521 Prague, Czech Republic

144 Department of Ethology, Institute of Animal Science, Přátelství 815, Prague, Uhříněves, 10400 ,

15 Czech Republic

165 Department of Game Management and Wildlife Biology, Faculty of Forestry and Wood Sciences,

17 Czech University of Life Sciences Prague, Kamýcká 129, 16521 Prague 6, Czech Republic

186 Department of Zoology, Faculty of Sciences, University of South Bohemia, Branišovská 31a, České

19 Budějovice, 370 05, Czech Republic

207 Department of Ecology and Evolutionary Biology, University of California, 621 Young Drive South,

21 Los Angeles, CA 90095-1606, USA

228 Rocky Mountain Biological Laboratory, Box 516, Crested Butte, C0 81224, USA 


\section{Abstract}

1. Identity signals have been studied for over 50 years but there is no consensus as to how to quantify individuality. While there are a variety of different metrics to quantify individual identity, or individuality, these methods remain un-validated and the relationships between them unclear.

2. We contrasted three univariate and four multivariate metrics (and their different computational variants) and evaluated their performance on simulated and empirical datasets.

3. Of the metrics examined, Beecher's information statistic $\left(H_{S}\right)$ was the best one and could easily and reliably be converted into the commonly used discrimination score (and vice versa) after accounting for the number of individuals and calls per individual in a given dataset. Although Beecher's information statistic is not entirely independent of sampling parameters, this problem can be removed by reducing the number of parameters or by increasing the number of individuals.

4. Because it is easily calculated, has superior performance, can be used to describe single variables or signal as a whole, and because it tells us the maximum number of individuals that can be discriminated given a set of measurements, we recommend that individuality should be quantified using Beecher's information statistic.

Keywords: Individual recognition, Social behavior, Identity signal, Beecher's Information Statistic, Acoustic identification, Acoustic discrimination, Vocal individuality, Discriminant analysis 


\section{Introduction}

48 The fact that conspecific individuals differ in consistent ways underlies a number of theoretically important questions in biology such as explaining cooperative behavior or understanding the evolution of sociality (Crowley et al., 1996; Bradbury \& Vehrencamp, 1998; Tibbetts, 2004). Because it may be advantageous for animals to choose with whom they interact or respond to (Wilkinson, 1984; Godard, 1991), there may be selection both to produce individually-distinctive signals and to discriminate among them (Tibbetts \& Dale, 2007; Wiley, 2013). Individually-distinctive traits can also be used to help wildlife population censuses or to monitor individuals (Terry \& McGregor, 2002; Blumstein et al., 2011). For these purposes, identity information in animal signals has been quantified by several different univariate and multivariate metrics, especially in the acoustic domain (Miller, 1978; Hafner, Hamilton, Steiner, Thompson, \& Winn, 1979; Beecher, 1989; Searby \& Jouventin, 2004; Mathevon, Koralek, Weldele, Glickman, \& Theunissen, 2010).

For identity signals to function properly, they should maximize the between-individual variation and minimize the within-individual variation. Therefore, to quantify an individual's identity we require repeated measurements of one or more traits on a given set of individuals within a population. This is well acknowledged in the study of acoustic signals (e.g., Hutchison, Stevenson, \& Thorpe, 1968; Beecher, 1989; Robisson, Aubin, \& Bremond, 1993). A typical study of acoustic identity signaling would record large number of vocalizations from each individual under different conditions (different time intervals, distances, etc.), measure a set of acoustic traits (e.g., fundamental frequency, duration, formant structure, frequency modulation, etc.), and then calculate the individual identity either directly through comparing between and within individual variation, or indirectly through discrimination between individuals. In studies of chemical or visual signals, robust assessment of within-individual variation by having many replicates from a single individual remains uncommon (Kondo \& Izawa, 2014; but see, e.g., Kean, Chadwick, \& Müller, 2015) although quantification of individual identity might be expected in future studies. 
A variety of identity metrics have proliferated because the existing metrics were considered biased (Beecher, 1989; Mathevon et al., 2010) or unsuitable for a particular signal type (Searby \& Jouventin, 2004). Furthermore, different equations have been sometimes used to calculate the same identity metric (Beecher, 1989; Lein, 2008; Charrier, Aubin, \& Mathevon, 2010; Linhart \& Šálek, 2017). Thus there is no consensus about how to properly measure identity. As a result, researchers have generally avoided quantitative comparisons between studies (Insley, Phillips, \& Charrier, 2003), although there have been a few of using exactly the same methods for several different species (Beecher, Medvin, Stoddard, \& Loesche, 1986; Lengagne, Lauga, \& Jouventin, 1997; Pollard \& Blumstein, 2011). The lack of a commonly used identity metric is a major impediment toward understanding the evolution of identity signaling and indeed, the evolution of individuality.

Here we review previously developed univariate and multivariate metrics that have been used to quantify individual identity information in signals and we test their performance on simulated and empirical datasets. In particular, we investigated the following metrics: F-value, Potential of individual coding PIC, Beecher's information statistic $H_{S}$, Efficiency of modulated signature $H_{M}$, and Mutual information MI. We further evaluated different computational variants found in literature in case of PIC and $\mathrm{H}_{\mathrm{S}}$ (see Methods and Supplement 1 for a detail overview of metrics and their variants).

We compare the performance of metrics to a hypothetical ideal identity information metric. We propose that ideal identity metric should have two basic characteristics: 1) it should not be systematically biased by study design (no systematic effects of number of individuals in a study and number of calls per individual in a study); and 2) in the multivariate case (i.e., when it is used to quantify individuality based on measurements of multiple signal features), it should rise with number of meaningful parameters and decrease with covariance between them. Also, for both univariate and multivariate case, we expect the metric will have a meaningful zero in case there is no identity content in a signal. Finally, we expect no upper limit on the degree of individuality; in theory, and 
97 given sufficient variation and variables, one could discriminate among an infinite number of

98 individuals. We also wished to see if each of two commonly used metrics (Beecher's information

99 statistic $\mathrm{H}_{s}$, and discrimination score DS) could be converted to the other metric to facilitate

100 comparative analyses of the evolution of individuality.

\section{Material and methods}

We used R for simulations and statistical analysis (R Core Team, 2012). Our simulated and empirical data along with analysis scripts are available on GitHub (Linhart, 2018).

105 Simulated datasets. We constructed datasets with univariate and multivariate normal distributions with parameters covering wide range of values - individuality (id $=0.01,1,2.5,5,10$ ), number of observations / calls per individual $(0=4,8,12,16,20)$, number of individuals $(i=5,10,15,20,25,30$, 35,40 ), and, for multivariate datasets, the covariance among variables (cov $=0,0.25,0.5,0.75,1$ ) and the number of variables $(p=2,4,6,8,10)$. Individuality (id) represents ratio of standard deviations between and within individuals (id $=S D_{\text {between }} / S D_{\text {within }}$; $S D_{\text {between }}$ was calculated from means for each individual). A single covariance (cov) value was used in the variance-covariance matrix to define covariances between all pairs of variables (detailed description in Supplement 2). We asked how dataset parameters (i, o, p, cov, id) influenced the value of each identity metric. To explore this, all combinations of dataset parameters were exhaustively sampled with 20 iterations on each unique combination of parameters. In each iteration, a new dataset was generated to ensure independence between samples. We developed R scripts involving "rnorm" and MASS package 

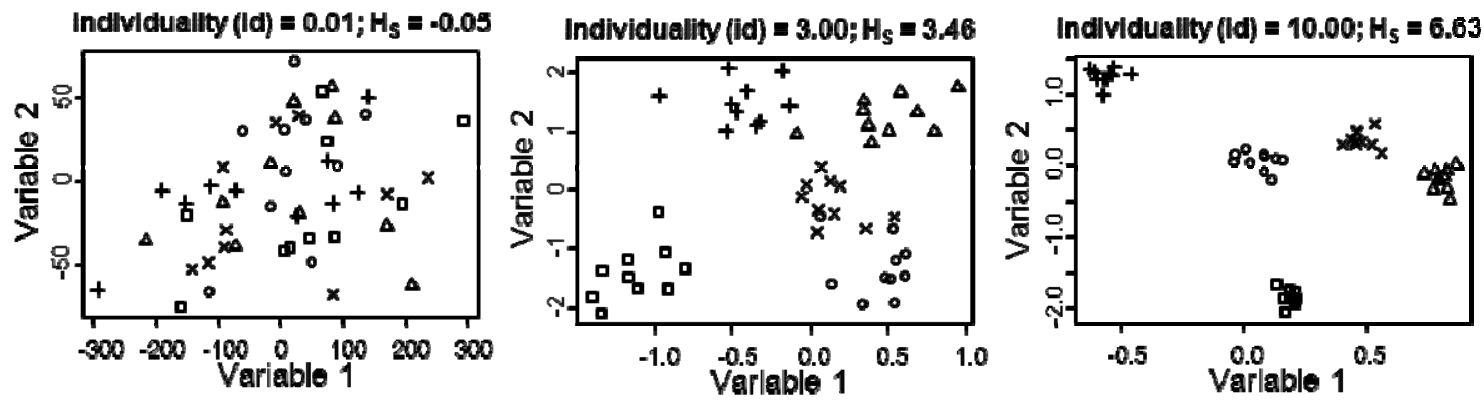

119

120

121

122

123

124

125

126

127

128

129

130

131

132

133

134

135

Figure 1. Illustration of three artificial multivariate datasets that differ only in the individuality used to generate datasets. Settings for the function generating these datasets: $i=5,0=10, p=2, \operatorname{cov}=0$, id $=0.01,3$, and 10

Empirical datasets. We used six datasets from four different species: little owls Athene noctua (ANmodulation, ANspec) (Linhart \& Šálek, 2017), corncrake Crex crex (CCformants, CCspec) (Budka \& Osiejuk, 2013), yellow-breasted boubous Laniarius atroflavus (LAhighweewoo) (Osiejuk et al. unpublished data), and domestic pigs Sus scrofa (SSgrunts) (Syrová, Policht, Linhart, \& Špinka, 2017) (Figure 2). In two species - corncrakes and little owls - calls were described by two different sets of variables. In little owls, we described calls by frequency modulation (ANmodulation) or parameters describing the distribution of the frequency spectrum (ANspec). In corncrakes, we used formants (CCformants) and parameters describing the distribution of the frequency spectrum (CCspec).

Because datasets varied with respect to the number of individuals ( $33-100)$ and the number of calls per individual available $(10-20)$, we scaled all datasets down to lowest common denominator by randomly selecting individuals and calls from bigger datasets. Eventually, each dataset had 33 individuals and 10 calls per individual. Each dataset also used different numbers of variables to describe the calls' acoustic structure (ANmodulation $=11$, ANspec $=7$, CCformants $=4$, CCspec $=7$, LAhighweewoo $=7$, SS grunty $=10$ ). In all these empirical datasets, assumptions of multivariate normality were tested (Korkmaz, Goksuluk, \& Zararsiz, 2014), but not met. This issue is common for research studies on acoustic individual identity. Authors deal with it by eliminating problematic variables (e.g., Sousa-Lima, Paglia, \& da Fonseca, 2008; Couchoux \& Dabelsteen, 2015), using non- 
140 Zuberbuehler, 2013), or by relying on robustness of cross-validated DFA towards relaxed

141 assumptions (e.g., Mathevon et al., 2010; Schneiderová, 2012). We used the last approach. If the

142 assumptions of discriminant analysis are not met the results should be less stable when using

143 different sampling and hence our results should be conservative.

144

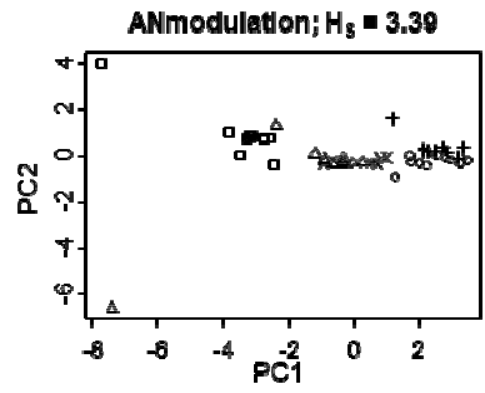

cCformants; $\mathrm{H}_{\mathrm{S}}=\mathbf{1 0 . 5 2}$

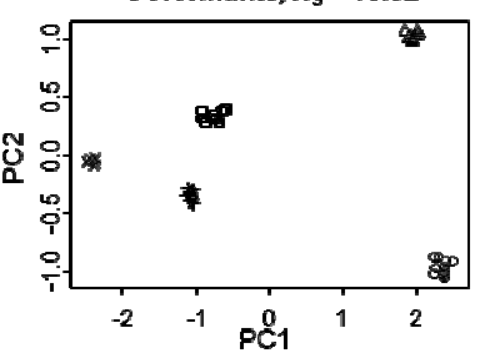

ANspec; $\mathrm{H}_{\mathrm{g}}=4.61$

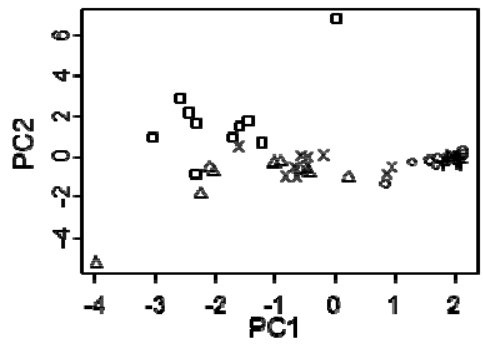

LAhlghweewoo; $\mathrm{H}_{\mathrm{S}}=\mathbf{3 . 5 7}$

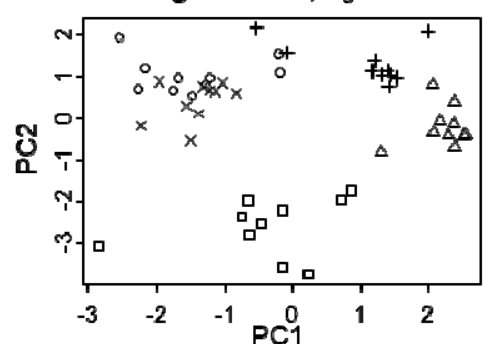

CCspec $; \mathrm{H}_{\mathrm{S}}=5.15$

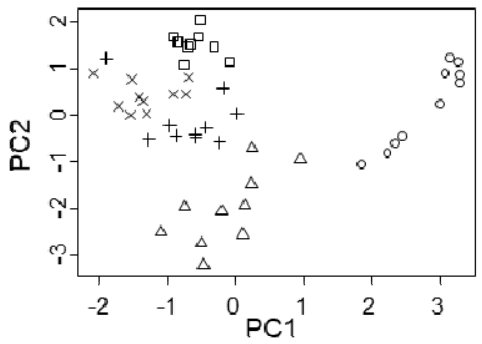

Ssgrunts; $H_{S}=2.57$

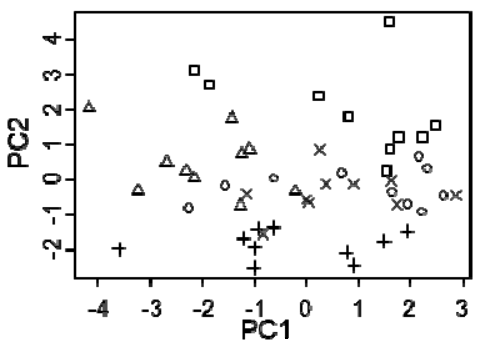

Figure 2. Illustration of empirical datasets. Five individuals were randomly sampled from each

dataset of 33 individuals and all 10 calls per individual were selected. $H_{s}$ for a full dataset is shown.

Data were centered and scaled and subjected to PCA. The first two Principal Components are

plotted.

The following scripts were used to calculate seven variants of three univariate metrics: $F$ value 


$$
P I C=\frac{C V_{b}}{C V_{w}}
$$

155 Two variants of PIC differ in whether $\mathrm{CV}_{\mathrm{b}}$ in the formula is calculated from all values (PIC $\mathrm{C}_{\text {betweentot }}$ )

156 (e.g., Charrier et al., 2010), or means for each individual are calculated first and $\mathrm{CV}_{\mathrm{b}}$ is then calculated

157 from these means ( $\mathrm{PIC}_{\text {betweenmeans }}$ ) (e.g., Lein, 2008). $\mathrm{H}_{\mathrm{S}}$ is based on F-value but unlike F-value, $\mathrm{H}_{\mathrm{S}}$ 158 accounts for sample size:

$$
H_{S}=\log _{2} \sqrt{\frac{F+n-1}{n}}
$$

The source of confusion is the ' $n$ ' in the formula. Total sample size $\left(H_{\text {Sntot }}\right)$, number of groups (i.e., individuals) $\left(H_{\text {Sngroups }}\right)$, and number of samples per group $\left(H_{\text {Snpergroup }}\right)$ could all be used as ' $n$ ' in this equation. Some studies explicitly state they used number of individuals as ' $n$ ' (e.g., Pollard, Blumstein, \& Griffin, 2010; Linhart \& Šálek, 2017), but the properties of $\mathrm{H}_{\mathrm{S}}$ values in these studies did not match the properties suggested in the original article by Beecher (1989). Yet another approach to calculate $\mathrm{H}_{\mathrm{S}}$ is to extract the variance component estimates and use the total (? ? $)$ and the residual variance ([? ${ }_{W}$, associated with random factor) to calculate $\mathrm{H}_{\mathrm{S}}\left(\mathrm{H}_{\text {svarcomp }}\right)$ (Beecher, 1989; Carter, Logsdon, Arnold, Menchaca, \& Medellin, 2012):

$$
H_{S}=\log _{2} \frac{\sigma_{T}}{\sigma_{W}}
$$

The following scripts were used to calculate multivariate metrics: calcDS, calcHSnpergroup, calcHM, calcMI. The calcDS is based on 'Ida' ('MASS' package). The calcMI function uses 'Ida' ('MASS' package) and 'mutinformation' ('infotheo' package).

Multivariate identity metrics were always calculated from data (simulated or empirical) that were centered to have a mean of zero, scaled to unit variance, and subjected to principal component analysis. 


\section{Statistical analysis}

175 Our goal was to ask whether there are systematic biases for each identity metric given different

176 parameters that reflect sampling design. The relationship between a given identity metric and each

177 of the parameters was assessed graphically by plotting the mean value and the $95 \%$ confidence

178 intervals of an identity metric against all of the modelled data parameters separately. We then used

179 a one-way ANOVA to test whether an identity metric was constant across all levels of a parameter. If

180 we found significant differences, we followed up these with post-hoc Tukey tests to identify which

181 parameter levels differed. Due to high number of comparisons, we only reported comparisons of

182 neighboring parameter levels. We used linear and non-parametric loess regression to convert $\mathrm{H}_{\mathrm{s}}$ to

183 DS and vice versa. Loess regression included the number of individuals and number of calls per

184 individual as additional predictors. We used Spearman correlation coefficients to quantify between-

185 metric consistency of ranking individuality in datasets. Pearson correlations were used to assess

186 consistency within identity metrics in full and partial datasets. We then used Friedman test, followed

187 by a series of Wilcoxon tests (for post-hoc comparison of differences between levels), to compare

188 correlation coefficients obtained for each pair of the metrics. 


\section{Results}

191 The comparison of available univariate and multivariate metrics to an ideal metric is shown in Table

1921.

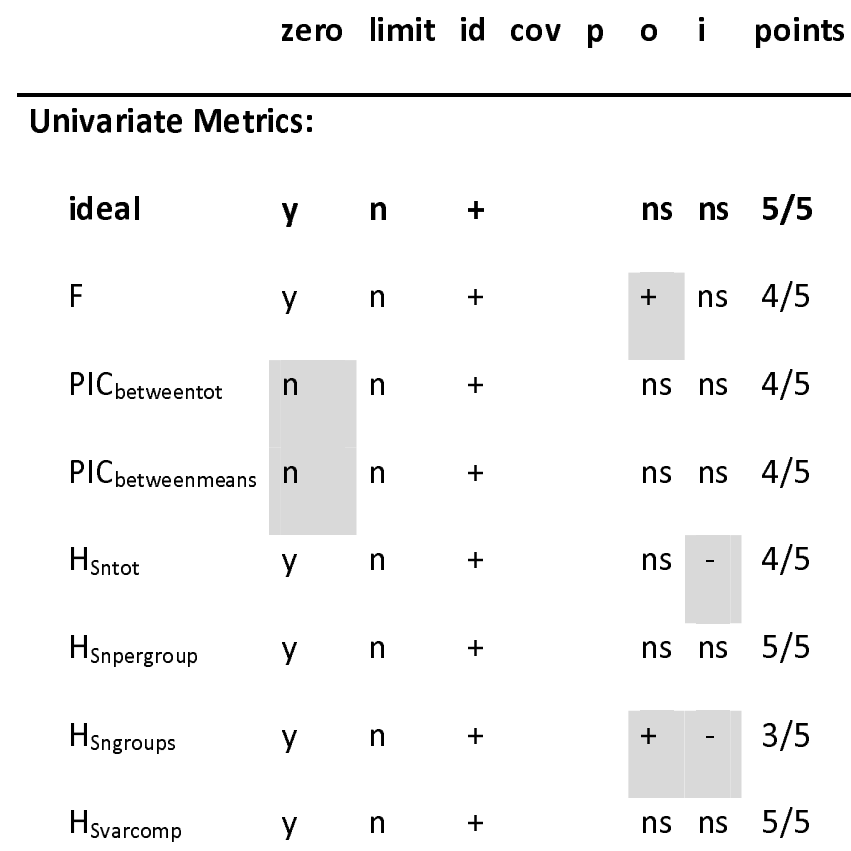

\section{Multivariate Metrics:}

\begin{tabular}{|c|c|c|c|c|c|c|c|c|}
\hline ideal & $y$ & $n$ & + & - & + & ns & ns & $7 / 7$ \\
\hline DS & $y$ & $y$ & + & - & + & + & - & $4 / 7$ \\
\hline $\mathrm{H}_{\mathrm{S}}$ & $y$ & $n$ & + & - & + & ns & + & $6 / 7$ \\
\hline $\mathrm{H}_{\mathrm{M}}$ & $y$ & $n$ & + & ns & ns & ns & ns & $5 / 7$ \\
\hline $\mathrm{MI}$ & $n$ & $y$ & + & - & + & - & + & $3 / 7$ \\
\hline
\end{tabular}

Table 1. The comparison of available univariate and multivariate metrics to a hypothetical ideal metric. We summed the number of matches (points) to compare different metrics to the ideal metric. Non-matching cells are highlighted in grey background. 'zero' - metric has a meaningful zero; 'limit' - metric is limited from the top by an asymptote; 'id' - change in response to increasing 
199 identity information in data; 'cov' - response to increasing covariance between variables; ' $p$ ' -

200 response to increasing number of variables; 'o' - response to increasing number of calls per

201 individual; 'i' - response to increasing number of individuals; 'y'- yes; 'n' - no; '+' - increase; '-' -

202 decrease; 'ns' - not significant, does not change with a parameter.

203 Univariate metrics

204 Univariate metrics: F, PIC variants ( IC $\left._{\text {betweentot, }} \mathrm{PIC}_{\text {betweenmeans }}\right), \mathrm{H}_{\mathrm{S}}$ variants $\left(\mathrm{H}_{\text {Sntot }}, \mathrm{H}_{\text {Snpergroup }}, \mathrm{H}_{\text {Sngroups }}\right.$

$\left.205 \mathrm{H}_{\text {Svarcomp}}\right)$.

206 All explored univariate metrics increased with increasing individuality in the data. However, only

$207 \mathrm{PIC}_{\text {betweentot}}, \mathrm{PIC}_{\text {betweenmeans, }} \mathrm{H}_{\text {Snpergroup }}$ and $\mathrm{H}_{\text {Svarcomp }}$ estimates were independent of the number of calls

208 and the number of individuals used to calculate the metric (Figure 3). These general patterns were

209 qualitatively identical when all results were pooled or if only one of the parameters (number of calls,

210 number of individuals, individuality) was changed at a time and the others were kept constant at the

211 middle value (see Supplement 3 for detailed results including ANOVA tests). 

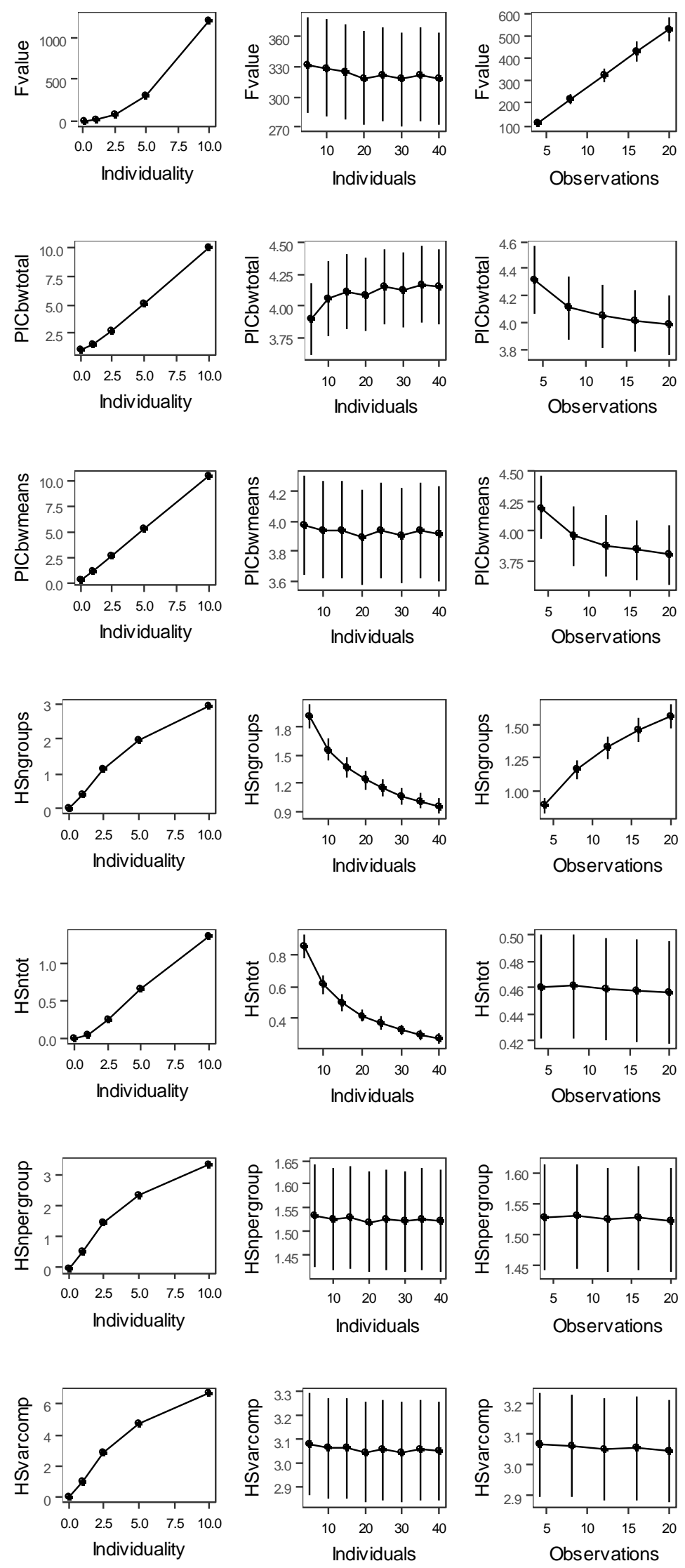
219 Figure 3. Variation in univariate identity metrics in response to artificial dataset parameters:

220 individuality, number of calls per individual, and number of individuals. Means and $95 \%$ confidence

221 intervals are shown. Graphs were plotted using all data pooled together.

222 Multivariate metrics

223 The performance of multivariate identity metrics is illustrated in Figure 4. All metrics increased with

224 increasing individuality. DS, $\mathrm{H}_{\mathrm{S}}$, and $\mathrm{Ml}$ increased with increasing number of variables available and

225 decreased with increasing covariance between variables. Only $\mathrm{H}_{\mathrm{M}}$ did not change in response to

226 increasing the number of individuals. $\mathrm{H}_{S}$ and $\mathrm{H}_{M}$ did not change in response to increasing the number

227 of calls per individual. These general patterns were qualitatively identical when all results were

228 pooled or if one parameter was changed at a time and others were kept constant at the middle value

229 (see Supplement 5 for detailed results including ANOVA tests).
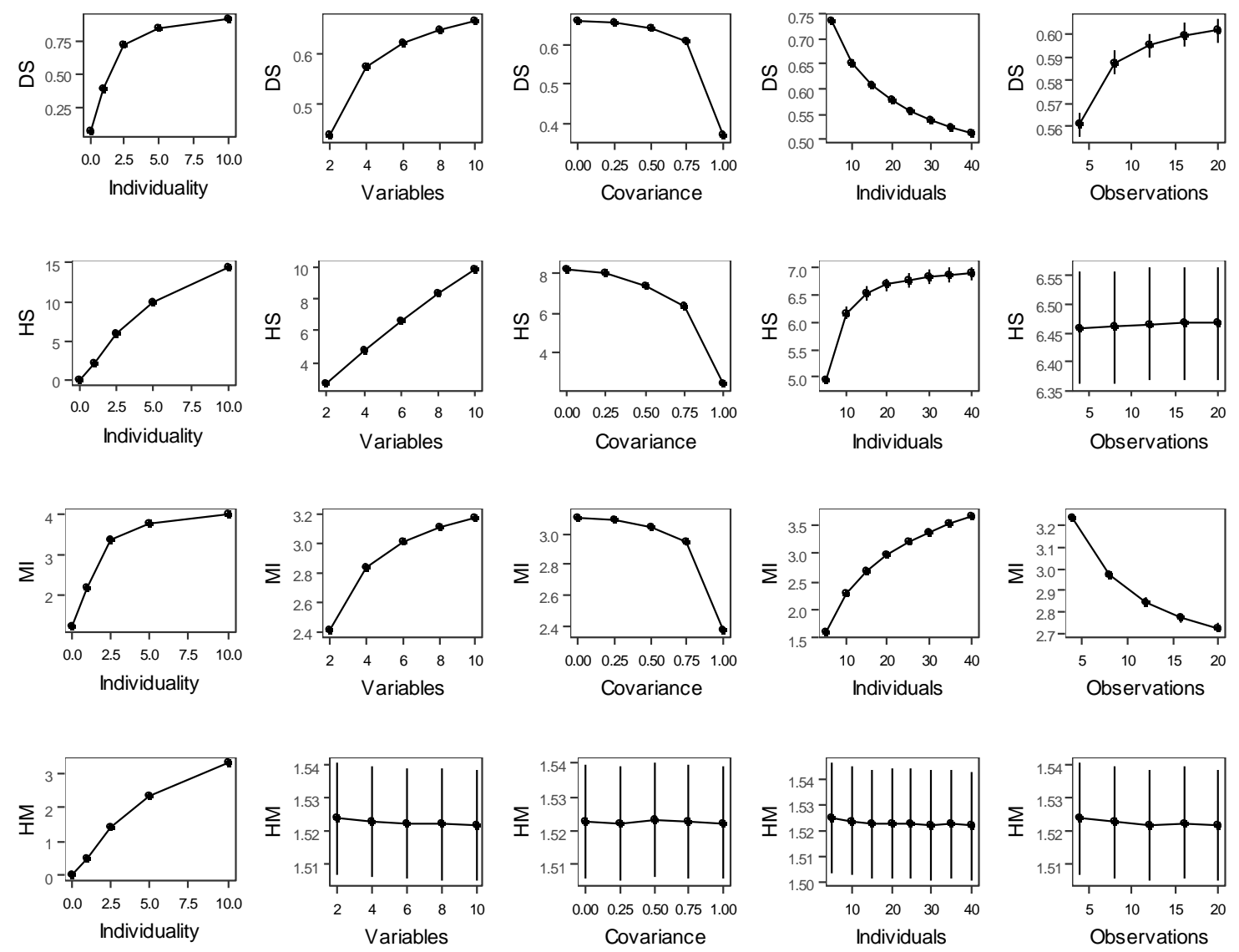
232 Figure 4. Multivariate identity metrics in response to changing individuality, covariance between 233 variables, number of variables, number of calls per individual, and number of individuals in artificial 234 data. Means and 95\% confidence intervals are shown. Despite the different response of metrics to some of the simulated parameters, there was still moderate to high agreement among metrics about identity content in the data (Spearman correlations, mean $r \pm S D=0.82 \pm 0.07$; minimum $r=0.71$ for correlation between $\mathrm{DS}$ and $\mathrm{Ml}$; maximum $r=0.95$ for correlation between DS and $\left.\mathrm{H}_{S}\right) . \mathrm{H}_{S}$ had the greatest correlations with other metrics (average $R=0.88$ ). We found no advantage to using $H_{M}$ over $H_{S}$ as previously suggested. Instead, $H_{M}$ was equal to $H_{S}$ per variable $\left(H_{M}=H_{S} / p\right)$ (Supplement 6). metric in 6/7 cases, followed by $\mathrm{H}_{\mathrm{M}}(5 / 7)$, DS (4/7), and MI (both 3/7) (Table 1 ).

243 Potential for removing bias in $\mathrm{H}_{S}$

244 We observed no significant association between $\mathrm{H}_{\mathrm{S}}$ and the number of individuals in the univariate case so the question arose about the precise cause of the bias in the multivariate case. This bias was only present when data were subjected to Principle Components Analysis (PCA). However, PCA is required to create uncorrelated components for $\mathrm{H}_{S}$ calculation. It is possible that the more variables measured, the more individuals need to be sampled in order to reduce this bias. We therefore fixed the number of variables to 5,10 , and $20(p=5,10,20)$ and varied the ratio of number of individuals to number of variables ' $i$ to $p$ ratio' from 0.5 to 5 (' $i$ to $p$ ratio' $=0.5,1,1.5,2,3,5$ ) by using different numbers of individuals in our simulations $(i=3,5,8,10,15,20,25,30,40,50,60,100$ depending on number of variables and " $i$ to $p$ ratio"). The number of calls per individual was set to 10 . Individuality and covariance were both chosen randomly in each iteration from predefined intervals used in the earlier simulations (covariance range $=[0,0.25,0.5,0.75,1]$; individuality range $=[0.01,1,2.5,5$, 10]). We used 100 and 1000 iterations for each ' $\mathrm{i}$ to $\mathrm{p}$ ratio' to get less and more conservative estimates. $\mathrm{H}_{S}$ did not rise significantly after the number of individuals reached at least the number of 
differences between levels if ' $i$ to $p$ ' $\geq 1$, all $p>0.132$ ) (Figure 5), or at least twice the number of

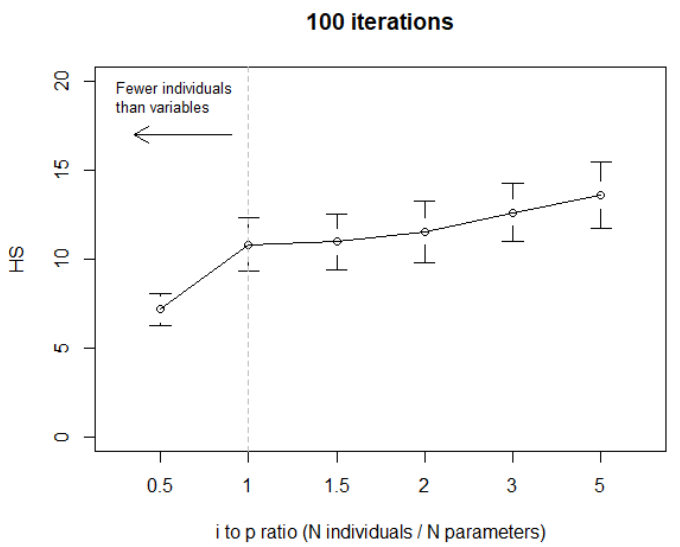

262 Figure 5. $\mathrm{H}_{\mathrm{S}}$ and " $\mathrm{i}$ to $\mathrm{p}$ ratio" (number of individuals / number of variables) for situation with 100

263 iterations. $H_{S}$ was under-estimated if there are fewer individuals than variables. Means and $95 \%$

264 confidence intervals are shown.

\section{Converting $\mathrm{DS}$ to $\mathrm{H}_{\mathrm{S}}$ and vice versa}

266 We used simple linear regression and non-parametric loess regression to estimate $\mathrm{H}_{S}$ based on DS

267 and vice versa. There was a previously suggested linear relationship that had a limit of $\mathrm{H}_{S}=8$ where

268 the DS values were $100 \%$ correct discrimination (Beecher 1989). Because the $\mathrm{H}_{\mathrm{S}}$ values in our original

269 simulated datasets far exceeded 8 in many cases (maximum $\mathrm{H}_{\mathrm{S}}=32.9$ ), we generated a new set of

270 simulated datasets with individuality ranging between 0.1 and 2 (id $=0.1,0.25,0.5,0.75,1,1.33$,

$2711.66,2)$, covariance set to zero (cov =0), number of iterations was reduced to 10 (it $=10)$, and other

272 parameters were set as in previous models $(p=2,4,6,8,10 ; i=5,10,15,20,25,30,35,40 ; o=4,8$,

$27312,16,20)$. These settings led to $H_{S}$ values up to 13.0 for data used for model building, and $H_{S}$ values

274 up to 14.4 in the case of data used for model testing. These values are much closer to 8 and also

275 much closer to $\mathrm{H}_{\mathrm{S}}$ values reported from nature. 
277 predictors the number of calls per individual and the number of individuals. We compared the loess

278 conversion and linear conversion models of DS and $\mathrm{H}_{\mathrm{s}}$. In general, loess estimates were closer to the

279 ideal prediction (intercept $=0$, beta $=1$ ) and the loess model reduced error of both DS and $\mathrm{H}_{S}$

estimates to about a half compared to linear estimates (Figure 6). Both $\mathrm{H}_{\mathrm{S}}$ estimates were

underestimated for high values of $H_{S}$. The ceiling value is clearly apparent for linear estimates of $H_{S}$. It
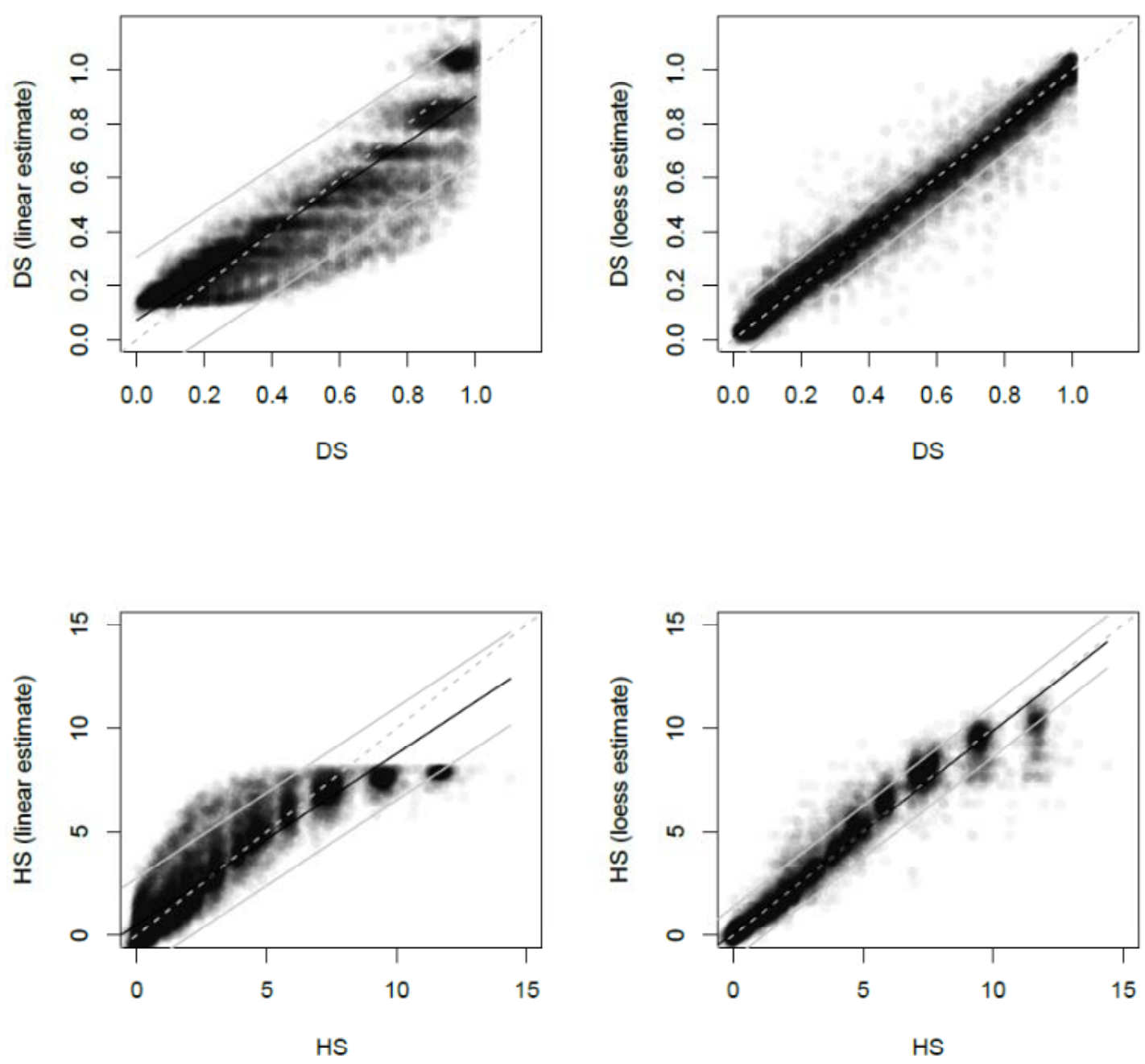

Figure 6. Estimation of $H_{S}$ and DS based on linear and loess transformation of DS and $H_{S}$ respectively 
286 for datasets with $\mathrm{H}_{S}$ up to 14.4. Linear DS estimation: Intercept $=0.07$, Beta $=0,83, \mathrm{R}^{2}=0.83$,

287 Standard Error of Estimate $(\mathrm{SEE})=0.12,95 \%$ Prediction interval $=$ predicted value \pm 0.23 ; DS loess

288 estimation: Intercept $=0.01$, Beta $=0.98, R^{2}=0.97$, Standard Error of Estimate $(S E E)=0.05,95 \%$

289 Prediction interval $=$ predicted value \pm 0.10 . Linear $H_{s}$ estimation: Intercept $=0.51$, Beta $=0.83, R^{2}=$

2900.83 , Standard Error of Estimate $(S E E)=1.14,95 \%$ Prediction interval = predicted value $\pm 2.24 ; \mathrm{HS}$

291 loess estimation: Intercept $=0.11$, Beta $=0.98, R^{2}=0.95$, Standard Error of Estimate $(\mathrm{SEE})=0.64$,

$29295 \%$ Prediction interval $=$ predicted value \pm 1.26

293

294

295

296

297

298

299

300

301

302

303

304

305

306

307

308

309

310

\section{Correlations between calculated and estimated metrics}

We were further interested in how $\mathrm{H}_{\text {sest }}$ and $D S_{\text {est }}$ might represent $\mathrm{H}_{S}$ and DS of a particular sample of individuals or $\mathrm{H}_{\text {sfull }}$ and $\mathrm{DS}$ full of the whole population. For this purpose, we first generated 50 full datasets with different identity levels representing 50 hypothetical populations of different species.

Each dataset comprised of 40 individuals, 20 calls per individual, and 10 parameters. For these datasets, individuality was set randomly ranging between $0.2-2$ ( 0.1 increments), and the covariance was set randomly ranging between $0.2-0.8$ (0.1 increments). These settings generated datasets with $\mathrm{H}_{\text {sfull }}$ values that ranged from $0.22-9.89$ (mean $\pm s d: 4.72 \pm 2.95$ ). Then, we repeatedly subsampled these datasets to get partial datasets which simulate different sampling of the population. We subsampled 5-40 individuals and 4-20 calls per individual per dataset in each of total 20 iterations. We also repeatedly subsampled our empirical datasets. We subsampled 5-33 individuals and $4-10$ calls per individual per dataset in each of total 20 iterations. The number of parameters was not randomized - we always kept the original number of variables.

In simulated datasets, $\mathrm{H}_{\mathrm{s}}$ and $\mathrm{H}_{\text {sest }}$ were correlated almost perfectly with each other and with $H_{\text {sfull }}$ (all average Pearson $r>0.97$ ). There was no difference among correlation coefficients from correlations between $\mathrm{H}_{\text {sfull, }}, \mathrm{H}_{\mathrm{S}}$, and $\mathrm{H}_{\text {Sest }}($ Friedman Chi Square $=3.6, \mathrm{p}=0.165)$. In empirical datasets, $H_{S}$ calculated on partial datasets still reflected the $H_{\text {sfull }}$ almost perfectly (average Pearson $r=0.99$ ). While $\mathrm{H}_{\text {sest }}$ reflected $\mathrm{H}_{\mathrm{S}}$ of partial dataset (average Pearson $r=0.90$ ), and $\mathrm{H}_{\text {sfull }}$ (average Pearson $r=$ 
3110.88 ) was slightly worse, it remained a reasonable fit. However, $\mathrm{H}_{\text {Sest }}$ did not reflect $\mathrm{H}_{\text {sfull }}$ as precisely

312 as it did $H_{s}$ (Friedman Chi Square $=33.6, p<0.001$, post-hoc test: $H_{s}-H_{\text {sfull }}$ vs. $\left.H_{\text {sest }}-H_{\text {sfull, }} p<0.001\right)$.

313 DS in simulated datasets, was almost perfectly correlated with $\mathrm{DS}_{\text {est }}$ (average Pearson $r=0.99$ ).

314 Although the relationship between DS and $\mathrm{DS}_{\text {est }}$ was significantly worse in a full dataset (DS full)

315 (Friedman Chi Square $=40.0, p<0.001$; both post-hoc tests: $p<0.005$ ), these associations remained

316 strong (DS full and DS: average Pearson $r=0.95 ; D_{\text {full }}$ and $D_{\text {est }}$ : average Pearson $\left.r=0.96\right)$. In empirical

317 datasets, the correlation between DS and $\mathrm{DS}_{\text {est }}$ was lower than in case of artificial datasets (average

318 Pearson $r=0.91$ ). DS and $D S_{\text {est }}$ of partial datasets had comparable correlations to $D S_{\text {full }}\left(D S_{\text {full }}\right.$ and $D S$ :

319 average Pearson $r=0.88$; $D S_{\text {full }}$ and $D S_{\text {est }}$ : average Pearson $\left.r=0.86\right)$. Thus, the performance of $D S$ and

$320 D_{\text {est }}$ to reflect each other or DS full did not differ (Friedman Chi Squre $=0.9, p=0.638$ ).

\section{Discussion}

322 All identity metrics had systematic biases that emerged from sampling decisions. Biases induced by

the number of individuals and the number of calls per individual in a sample both decreased with

$\mathrm{H}_{\mathrm{S}}$ could be converted reliably into DS and vice-versa.

Beecher, 1989) and Potential for individual coding (PIC) (Robisson et al., 1993; Lengagne et al., 1997) 
336 way as $2^{H_{S}}$ does. However, PIC slightly differs from $\mathrm{H}_{\mathrm{s}}$ and deviates from expected zero values if there

337 is low identity content in a signal that approaches zero. It is important to realize that variables with

$338 \quad \mathrm{PIC}_{\text {betweentot }}$ value $>1$ need not convey meaningful individual information as commonly assumed.

339 Using the PIC betweentot does not create overly spurious conclusions but rather including more less-

340 important variables increases noise in subsequent analyses. Studies using the number of individuals

341 as ' $n$ ' to calculate $H_{S}$ most likely under-estimates the real $H_{S}$ value because the number of individuals

342 is typically higher than the number of calls per individual in those studies. $\mathrm{H}_{\mathrm{s}}$ has been suggested as a

343 suitable metric for comparative analyses and $\mathrm{H}_{\mathrm{S}}$ has been used for such purposes in a few such

344 analyses. We think the overall conclusions of these analyses are valid whenever the same sampling

345 protocol was used across species (e.g., Pollard \& Blumstein, 2011).

identity metric, despite numerous studies showing systematic biases in DS (e.g., Beecher, 1989; Bee, exceed the performance of $\mathrm{H}_{\mathrm{s}}$ (Searby \& Jouventin, 2004; Mathevon et al., 2010) despite the fact that both are grounded in information theory and use the same measurement unit (bits) as $\mathrm{H}_{s}$. The robustness of $H_{M}$ towards sampling reported here (number of individuals, number of calls, even number of variables and covariance) could be seen as attractive. However, as we show, $\mathrm{H}_{M}$ quantifies identity information per variable and not the identity information of the entire signal. If one is interested in total identity information, with $\mathrm{H}_{M}$, it is necessary to know the effective number of variables (i.e., if there is perfect covariance between the variables, the effective number of variables 
362 similar shortcomings as discrimination scores. Our results showing biases in MI are in line with

363 previous studies that investigated measures of clustering for various machine learning purposes

364 where potentially unbiased variants of MI are searched for (Marrelec, Messé, \& Bellec, 2015; Amelio

$365 \&$ Pizzuti, 2017).

$366 \quad$ Although we suggest that $\mathrm{H}_{\mathrm{S}}$ should be generally used to quantify individuality, some

367 questions on identity signaling might still need to rely on the other identity metrics or approaches.

368 For example, researchers might be interested in whether distinctiveness of individuals increases

369 during ontogeny (Briefer \& McElligott 2012, Lapshina et al., 2012, Syrová et al., 2017). In such cases,

370 assessment on individual level is required (distances, discrimination score) while $\mathrm{H}_{\mathrm{s}}$ would only

371 provide overall identity information for each ontogeny stage making further statistical assessment

372 impossible.

Precision of conversion between metrics. Both $H_{S}$ and $H_{M}$ values were previously found to correlate well with DS (Beecher, 1989; Searby \& Jouventin, 2004). We extend these previous findings on $H_{S}$ (Beecher, 1989) to situations with unequal sampling and we show it is possible to convert between $\mathrm{H}_{\mathrm{S}}$ and DS with an acceptable amount of error even when datasets differ in the number of individuals and calls per individual. Predicting DS from $\mathrm{H}_{\mathrm{S}}$ has an advantage of being more precise than predicting $\mathrm{H}_{S}$ from DS. The precision of conversion decreased in real datasets compared to simulated datasets. However, the decrease was not dramatic, especially when considering that the conversion model was derived from simulated datasets with only two uncorrelated variables while real datasets differed in both the number of variables and their covariance structure. Furthermore, real datasets had issues associated with multivariate normality, which is a common problem of many studies and which also likely worsened the conversion precision and metric consistency. 
387 individuals or species because our $\mathrm{H}_{s}$ and DS values based on an entire population or subsamples

388 from these populations were well correlated for both simulated and empirical datasets.

Sample size considerations. Biases of both DS and $\mathrm{H}_{\mathrm{S}}$ decrease with increasing sample sizes.

Researchers using DS as an identity metric have been warned about the problems with low sample acoustic modality only. It is increasingly recognized that signals may employ multiple modalities (Partan \& Marler, 1999; Proops, McComb, \& Reby, 2009; Pitcher, Briefer, Baciadonna, \& McElligott, 2017). There is no reason to believe that modality constrains the use of these metrics and, in 402 principle, all of the identity metrics could be used in visual or chemical domains as well (Beecher, 1982; Beecher, 1989; Kondo \& Izawa, 2014). However, identity information outside the acoustic 404 domain is rarely quantified with the metrics described here because they all require assessment of a signal's within individual variation. The reasons might be that other modalities are assumed to be more static or because of technical difficulties in quantifying within-individual variation. The latter seems to be a case. The latest progress in machine learning and image analysis suggests that it should be possible to conduct individual discrimination tasks in a similar way to that used for acoustic signals (Allen \& Higham, 2015; Van Belleghem et al., 2018). Finally, repeated sampling of individual 
411 \& Mills, 2018). Thus, researchers may try to quantify potential individual identity information in

412 visual and chemical signals in future studies.

413 Conclusion. We have shown that $\mathrm{H}_{\mathrm{S}}$ is the identity metric with the best performance in both

414 univariate and multivariate contexts. Given that $\mathrm{H}_{S}$ may not be sufficient in all cases, we encourage

415 further research to develop new metrics to quantify identity information in signals. However, new

416 metrics should always be appropriately assessed and their performance directly compared to the

417 best existing metrics. The datasets and algorithms we have provided should aid in future

418 comparisons.

\section{Acknowledgements}

420 PL received funding from the European Union's Horizon 2020 research and innovation programme

421 under the Marie Skłodowska-Curie grant agreement No. 665778 administered by the National

422 Science Centre, Poland (UMO-2015/19/P/NZ8/02507). DTB is supported by the NSF. MŠp, MS, and RP

423 were supported by Czech Science Foundation (GA14-27925S) and Czech Ministry of Agriculture (MZE-

424 RO0718). MŠá work was supported by the research aim of the Czech Academy of Sciences (RVO

425 68081766).

\section{Data Accessibility statement}

427 Data and code used for this article are available at GitHub and ZENODO public repositories under

428 permissive free software MIT license (Linhart 2018).

429

430

431

432

433

434

435

\section{References}

Allen, W. L., \& Higham, J. P. (2015). Assessing the potential information content of multicomponent visual signals: a machine learning approach. Proceedings of the Royal Society B-Biological Sciences, 282(1802), 20142284. doi:10.1098/rspb.2014.2284

Amelio, A., \& Pizzuti, C. (2017). Correction for closeness: Adjusting normalized mutual information measure for clustering comparison. Computational Intelligence, 33(3), 579-601.

doi:10.1111/coin.12100 
436 Bee, M. A., Kozich, C. E., Blackwell, K. J., \& Gerhardt, H. C. (2001). Individual variation in

437 advertisement calls of territorial male green frogs, Rana clamitans: Implications for individual

438 discrimination. Ethology, 107, 65-84. doi:10.1046/j.1439-0310.2001.00640.x

439 Beecher, M. D.(1982). Signature systems and kin recognition. American Zoologist, 22(3), 477-490.

440 Beecher, M. D., Medvin, M. B., Stoddard, P. K., \& Loesche, P. (1986). Acoustic adaptations for parent$441 \quad$ offspring recognition in swallows. Experimental Biology, 45, 179-193.

442 Beecher, M. D. (1989). Signaling systems for individual recognition - an information-theory approach. Animal Behaviour, 38, 248-261. doi:10.1016/S0003-3472(89)80087-9

Blumstein, D. T., Mennill, D. J., Clemins, P., Girod, L., Yao, K., Patricelli, G., ... Kirschel, A. N. G. (2011). Acoustic monitoring in terrestrial environments using microphone arrays: applications, technological considerations and prospectus. Journal of Applied Ecology, 48(3), 758-767. doi:10.1111/j.1365-2664.2011.01993.x

Bradbury, J. W., \& Vehrencamp, S. L. (1998). Principles of animal communication (1st ed.). Sunderland, MA: Sinauer Associates.

Briefer, E. F., \& McElligott, A. G. (2012). Social effects on vocal ontogeny in an ungulate, the goat, Capra hircus. Animal Behaviour, 83(4), 991-1000. doi:10.1016/j.anbehav.2012.01.020

Budka, M., \& Osiejuk, T. S. (2013). Formant frequencies are acoustic cues to caller discrimination and are a weak indicator of the body size of corncrake males. Ethology, 119, 960-969.

Budka, M., Wojas, L., \& Osiejuk, T. S. (2015). Is it possible to acoustically identify individuals within a doi:10.1111/eth.12141

457 Carter, G. G., Logsdon, R., Arnold, B. D., Menchaca, A., \& Medellin, R. A. (2012). Adult vampire bats produce contact calls when isolated: Acoustic variation by species, population, colony, and individual. Plos One, 7. doi:10.1371/journal.pone.0038791 
460 Charrier, I., Aubin, T., \& Mathevon, N. (2010). Mother-calf vocal communication in Atlantic walrus: a

461 first field experimental study. Animal Cognition, 13, 471-482. doi:10.1007/s10071-009-0298-

4629

463 Couchoux, C., \& Dabelsteen, T. (2015). Acoustic cues to individual identity in the rattle calls of

464 common blackbirds: a potential for individual recognition through multi-syllabic vocalisations

465 emitted in both territorial and alarm contexts. Behaviour, 152(1), 57-82.

466 doi:10.1163/1568539X-00003232

467 Crowley, P. H., Provencher, L., Sloane, S., Dugatkin, L. A., Spohn, B., Rogers, L., \& Alfieri, M. (1996).

468 Evolving cooperation: the role of individual recognition. Biosystems, 37(1), 49-66.

469 doi:10.1016/0303-2647(95)01546-9

470 Deshpande, K., Furton, K. G., \& Mills, D. E. K. (2018). The Equine volatilome: Volatile organic

471 compounds as discriminatory markers. Journal of Equine Veterinary Science, 62, 47-53.

472 doi:10.1016/j.jevs.2017.05.013

473 Godard, R. (1991). Long-term memory of individual neighbors in a migratory songbird. Nature,

$474 \quad 350(6315), 228-229$.

475 Hafner, G. W., Hamilton, C. L., Steiner, W. W., Thompson, T. J., \& Winn, H. E. (1979). Signature

476 information in the song of the humpback whale. Journal of the Acoustical Society of America,

$477 \quad 66,1-6$. doi:10.1121/1.383072

478 Hutchison, R. E., Stevenson, J. G., \& Thorpe, W. H. (1968). The basis for individual recognition by

479 voice in the Sandwich tern (Sterna sandvicensis). Behaviour, 32(1/3), 150-157.

480 Insley, S. J., Phillips, A., \& Charrier, I. (2003). A review of social recognition in pinnipeds. Aquatic

$481 \quad$ Mammals, 29, 181-201.

482 Kean, E. F., Chadwick, E. A., \& Müller, C. T. (2015). Scent signals individual identity and country of

483 origin in otters. Mammalian Biology - Zeitschrift Für Säugetierkunde, 80(2), 99-105.

484 doi:10.1016/j.mambio.2014.12.004 
Kondo, N., \& Izawa, E. (2014). Individual differences in facial configuration in large-billed crows. Acta Ethologica, 17(1), 37-45. doi:10.1007/s10211-013-0156-2

Korkmaz, S., Goksuluk, D., \& Zararsiz, G. (2014). MVN: An R package for assessing multivariate normality. The R Journal, 6(2), 151-162.

Lapshina, E. N., Volodin, I. A., Volodina, E. V., Frey, R., Efremova, K. O., \& Soldatova, N. V. (2012). The ontogeny of acoustic individuality in the nasal calls of captive goitred gazelles, Gazella subgutturosa. Behavioural Processes, 90, 323-330. doi:10.1016/j.beproc.2012.03.011

Lein, M. R. (2008). Song variation in Buff-breasted Flycatchers (Empidonax fulvifrons). Wilson Journal of Ornithology, 120, 256-267. doi:10.1676/07-067.1

Lengagne, T., Lauga, J., \& Jouventin, P. (1997). A method of independent time and frequency decomposition of bioacoustic signals: inter-individual recognition in four species of penguins. Comptes Rendus De L Academie Des Sciences Serie lii-Sciences De La Vie-Life Sciences, 320, 885-891. doi:10.1016/s0764-4469(97)80873-6

Linhart, P. (2018). pygmy83/Identity-metrics: Identity metrics. Zenodo. doi:10.5281/zenodo.1252271 Linhart, P., \& Šálek, M. (2017). The assessment of biases in the acoustic discrimination of individuals. PLOS ONE, 12(5), e0177206. doi:10.1371/journal.pone.0177206

Marrelec, G., Messé, A., \& Bellec, P. (2015). A bayesian alternative to mutual Information for the hierarchical clustering of dependent random variables. PLOS ONE, 10(9). doi:10.1371/journal.pone.0137278

Mathevon, N., Koralek, A., Weldele, M., Glickman, S. E., \& Theunissen, F. E. (2010). What the hyena's laugh tells: Sex, age, dominance and individual signature in the giggling call of Crocuta crocuta. BMC Ecology, 10, 9-Article No.: 9. doi:10.1186/1472-6785-10-9

McGarigal, K., Cushman, S., \& Stafford, S. (2000). Multivariate Statistics for Wildlife and Ecology Research. New York: Springer-Verlag. 
Mielke, A., \& Zuberbuehler, K. (2013). A method for automated individual, species and call type recognition in free-ranging animals. Animal Behaviour, 86(2), 475-482. doi:10.1016/j.anbehav.2013.04.017

Miller, D. B. (1978). Species-typical and individually distinctive acoustic features of crow calls of red jungle fowl. Zeitschrift Fur Tierpsychologie-Journal of Comparative Ethology, 47, 182-193.

Mundry, R., \& Sommer, C. (2007). Discriminant function analysis with nonindependent data: consequences and an alternative. Animal Behaviour, 74(4), 965-976. doi:10.1016/j.anbehav.2006.12.028

Partan, S., \& Marler, P. (1999). Communication goes multimodal. Science, 283(5406), 1272-1273. doi:10.1126/science.283.5406.1272

Pitcher, B. J., Briefer, E. F., Baciadonna, L., \& McElligott, A. G. (2017). Cross-modal recognition of familiar conspecifics in goats. Royal Society Open Science, 4(2), 160346. doi:10.1098/rsos.160346

Pollard, K. A., \& Blumstein, D. T. (2011). Social group size predicts the evolution of individuality. Current Biology, 21(5), 413-417. doi:10.1016/j.cub.2011.01.051

Pollard, K. A., Blumstein, D. T., \& Griffin, S. C. (2010). Pre-screening acoustic and other natural signatures for use in noninvasive individual identification. Journal of Applied Ecology, 47(5), 1103-1109. doi:10.1111/j.1365-2664.2010.01851.x

Proops, L., McComb, K., \& Reby, D. (2009). Cross-modal individual recognition in domestic horses (Equus caballus). Proceedings of the National Academy of Sciences, 106(3), 947-951. doi:10.1073/pnas.0809127105

R Core Team. (2012). R: A language and environment for statistical computing. Vienna, Austria: $R$ Foundation for Statistical Computing. Retrieved from http://www.R-project.org/

Robisson, P., Aubin, T., \& Bremond, J. (1993). Individuality in the voice of the Emperor penguin Aptenodytes forsteri - Adaptation to a noisy environment. Ethology, 94(4), 279-290. 
534 Schneiderová, I. (2012). Frequency-modulated second elements of two-element alarm calls do not

535 enhance discrimination of callers in three Eurasian ground squirrels. Current Zoology, 58(5),

$536 \quad 749-757$.

537 Searby, A., \& Jouventin, P. (2004). How to measure information carried by a modulated vocal

538 signature? Journal of the Acoustical Society of America, 116, 3192-3198.

$539 \quad$ doi:10.1121/1.1775271

540 Sousa-Lima, R. S., Paglia, A. P., \& da Fonseca, G. A. B. (2008). Gender, age, and identity in the

541 isolation calls of Antillean manatees (Trichechus manatus manatus). Aquatic Mammals, 34,

542 109-122. doi:10.1578/am.34.1.2008.109

543 Syrová, M., Policht, R., Linhart, P., \& Špinka, M. (2017). Ontogeny of individual and litter identity

$544 \quad$ signaling in grunts of piglets. The Journal of the Acoustical Society of America, 142(5), 3116-

$545 \quad$ 3121. doi:10.1121/1.5010330

546 Terry, A. M. R., \& McGregor, P. K. (2002). Census and monitoring based on individually identifiable

547 vocalizations: the role of neural networks. Animal Conservation, 5, 103-111.

$548 \quad$ doi:10.1017/s1367943002002147

549 Tibbetts, E. A. (2004). Complex social behaviour can select for variability in visual features: a case

550 study in Polistes wasps. Proceedings of the Royal Society of London B: Biological Sciences,

$551 \quad 271(1551), 1955-1960$. doi:10.1098/rspb.2004.2784

552 Tibbetts, E., \& Dale, J. (2007). Individual recognition: it is good to be different. Trends in Ecology \&

553 Evolution, 22(10), 529-537. doi:10.1016/j.tree.2007.09.001

554 Tripovich, J. S., Rogers, T. L., Canfield, R., \& Arnould, J. P. Y. (2006). Individual variation in the pup

555 attraction call produced by female Australian fur seals during early lactation. Journal of the

556 Acoustical Society of America, 120(1), 502-509. doi:10.1121/1.2202864

557 Van Belleghem, S. M., Papa, R., Ortiz-Zuazaga, H., Hendrickx, F., Jiggins, C. D., Owen McMillan, W., \&

558 Counterman, B. A. (2018). patternize: An R package for quantifying colour pattern variation.

559 Methods in Ecology and Evolution, 9(2), 390-398. doi:10.1111/2041-210X.12853 
560 Venables, W. N., \& Ripley, B. D. (2002). Modern Applied Statistics with S (Fourth). New York: Springer.

$561 \quad$ Retrieved from http://www.stats.ox.ac.uk/pub/MASS4

562 Wiley, R. H. (2013). Specificity and multiplicity in the recognition of individuals: implications for the

563 evolution of social behaviour. Biological Reviews, 88(1), 179-195. doi:10.1111/j.1469-

$564 \quad 185 \times .2012 .00246 . x$

565 Wilkinson, G. S. (1984). Reciprocal food sharing in the vampire bat. Nature, 308(5955), 181-184.

566 doi:10.1038/308181a0 